\title{
Clique-transversal sets and weak 2-colorings in graphs of small maximum degree
}

\author{
Gábor Bacsó ${ }^{1}$ and Zsolt Tuza ${ }^{1,2}$ \\ ${ }^{1}$ Computer and Automation Institute, Hungarian Academy of Sciences, H-1111 Budapest, Kende u. 13-17, Hungary \\ ${ }^{2}$ Department of Computer Science, University of Pannonia, H-8200 Veszprém, Egyetem u. 10, Hungary
}

received December 31, 2008, accepted June 8, 2009.

A clique-transversal set in a graph is a subset of the vertices that meets all maximal complete subgraphs on at least two vertices. We prove that every connected graph of order $n$ and maximum degree three has a clique-transversal set of size $\lfloor 19 n / 30+2 / 15\rfloor$. This bound is tight, since $19 n / 30-1 / 15$ is a lower bound for infinitely many values of $n$. We also prove that the vertex set of any connected claw-free graph of maximum degree at most four, other than an odd cycle longer than three, can be partitioned into two clique-transversal sets. The proofs of both results yield polynomial-time algorithms that find corresponding solutions.

2000 Mathematics Subject Classification: 05C15, 05C69, 05C85

Keywords: clique-transversal set, weak coloring, clique coloring, cubic graph, claw-free graph, polynomial-time algorithm

\section{Introduction}

We consider finite, simple undirected graphs $G=(V, E)$, with vertex set $V$ and edge set $E$. The number of vertices will be denoted by $n$.

There are slight differences in the usage of the term 'clique' in graph theory. Throughout this paper, we use clique with the following restricted meaning: inclusion-wise maximal complete subgraph with at least two vertices. Hence, isolated vertices will not be called cliques, and maximality under inclusion will be required.

A clique-transversal set is a set $S \subseteq V$ that meets all cliques of $G$. The smallest cardinality of a cliquetransversal set in $G$, called clique-transversal number, is denoted by $\tau_{C}(G)$. A weak 2-coloring of $G$ is a mapping $\phi: V \rightarrow\{r, g\}$ (say red, green) such that both $\phi^{-1}(r)$ and $\phi^{-1}(g)$ are clique-transversal sets. If such $\phi$ exists, we say that $G$ is weakly 2-colorable.

This notion can be extended to weak $k$-coloring, also called $k$-clique-coloring in the literature, which assigns one of $k$ colors to each vertex in such a way that no clique is monochromatic. Equivalently, graph $G$ is weakly $k$-colorable if there exists a partition $V_{1} \cup \cdots \cup V_{k}=V$ such that no $V_{i}$ contains any cliques of $G$. The smallest nonnegative integer $k$ admitting a weak $k$-coloring will be denoted by $\chi_{C}(G)$.

\footnotetext{
${ }^{\dagger}$ Research supported by the Hungarian Scientific Research Fund, grant OTKA T-049613.

1365-8050 @ 2009 Discrete Mathematics and Theoretical Computer Science (DMTCS), Nancy, France
} 


\subsection{Some standard terminology}

For $d=3,4$ we denote by $\mathcal{G}_{d}$ the class of connected graphs of maximum degree at most $d$. The members of $\mathcal{G}_{3}$ are the connected subcubic graphs, and those 3-regular ones are called cubic. The degree of vertex $v$ will be denoted by $d(v)$. A vertex is simplicial if its neighbors are mutually adjacent.

Given a "forbidden" graph $F$, graph $G$ is called $F$-free if no induced subgraph of $G$ is isomorphic to $F$. In the cases of $F=K_{3}, F=K_{1,3}$, and $F=K_{4}-e$ (one edge removed from $K_{4}$ ), we use the standard terms triangle-free, claw-free, and diamond-free, respectively. A hole is a chordless cycle of length at least four.

\subsection{Results and history}

The graph invariant $\tau_{C}$ was introduced by Gallai [13] and then studied by various authors. The earliest published results deal with chordal graphs [22], relating $\tau_{C}$ with the number of vertices under the assumption that each edge is contained in some clique of given order. The case of $\tau_{C} \leq n / 2$ has been analyzed for line graphs and their complements [4], and some general bounds on $\tau_{C}$ appeared in [11].

It should be noted that if $G$ is triangle-free, then a set is a clique-transversal set of $G$ if and only if it meets all edges-i.e., it is a vertex cover-therefore $\tau_{C}(G)$ is equal to the number of vertices minus the independence number of $G$ in this case. This also means that the determination of $\tau_{C}$ is algorithmically hard, on many restricted classes of graphs. Structured hard classes with respect to $\tau_{C}$ can be found in [9, 14, 8], whereas polynomial-time algorithms for other classes are given in [7, 9, 16].

Liang et al. [17] proved the following estimates on the clique-transversal number.

1. Every connected cubic graph $G$ of order $n>4$ has $5 n / 14 \leq \tau_{C}(G) \leq 2 n / 3$.

2. There exist infinitely many cubic connected graphs with $\tau_{C}(G)=3 n / 5$.

The estimates of [1] were proved also in [21], and the graphs attaining the lower bound were characterized in both papers. On the other hand, it remained an open problem to determine a tight upper bound on $\tau_{C}(G)$ as a function of $n$. It has been explicitly raised in [17, page 114] whether $\lceil 3 n / 5\rceil$ is a valid upper bound. Here we disprove this guess and prove the following estimates.

Theorem 1 Consider the class $\mathcal{G}_{3}$ of connected subcubic graphs.

1. If $G \in \mathcal{G}_{3}$ of order $n$ is not cubic, or contains a triangle, then $\tau_{C}(G) \leq 19 n / 30+1 / 30$.

2. If $G \in \mathcal{G}_{3}$ of order $n$ is cubic and triangle-free, then $\tau_{C}(G) \leq 19 n / 30+2 / 15$.

These bounds are tight in the sense that there exist infinitely many $G \in \mathcal{G}_{3}$, say of order $n$, such that

3. $G$ is cubic and $\tau_{C}(G)=19 n / 30-1 / 15$.

4. $G$ is not cubic and $\tau_{C}(G)=19 n / 30-3 / 10$.

Moreover, clique-transversal sets of sizes guaranteed in Parts 1 and 2 can be found in polynomial time for any $G \in \mathcal{G}_{3}$.

Determining $\chi_{C}(G)$ is hard: to decide $\chi_{C}=2$ is NP-complete on 3-chromatic perfect graphs [15], and can be even harder: it is $\Sigma_{2}^{p}$-complete on unrestricted input graphs [19]. On the positive side, all planar graphs have $\chi_{C} \leq 3[20]$ and $\chi_{C}=2$ can be tested in polynomial time if the input is restricted to planar instances [15], hence $\chi_{C}$ on planar graphs can be determined efficiently. 
A necessary and sufficient condition for $\chi_{C} \leq k$ on line graphs was given in [4]. Moreover, claw-free perfect graphs are weakly 2-colorable [6]. It was erroneously stated in [17, page 114] that the upper bound $\tau_{C} \leq n / 2$ implies $\chi_{C} \leq 2$ for claw-free cubic graphs; later, however, in an unpublished manuscript the authors of [17] gave a proof for weak 2-colorability. Here we extend this latter result by dropping the condition of regularity and also weakening the condition on vertex degrees.

Theorem 2 Every connected claw-free graph of maximum degree at most four, other than an odd hole, is weakly 2-colorable. Moreover, a weak 2-coloring can be found in polynomial time.

These results are proved in Sections 2 and 3 , respectively. Some related problems are mentioned in the concluding section.

\section{Transversal sets}

In this section we prove Theorem 1 . Let us begin with the proof of tightness, and then proceed with the upper bounds.

Proof of Parts 3 and 4. Locke [18] constructed an infinite family of connected cubic triangle-free graphs with $n:=30 k+22$ vertices and independence number $11 k+8$. Thus, in every such graph $G$ we have

$$
\tau_{C}(G)=19 k+14=19(n-22) / 30+14=19 n / 30-1 / 15 .
$$

If a non-regular connected graph is needed, we omit just one non-cutting vertex. Denoting $n:=30 k+21$ we obtain

$$
\tau_{C}(G)=19 k+13=19(n-21) / 30+13=19 n / 30-3 / 10 .
$$

Proof of Parts 1 and 2. Let $G=(V, E)$ be a subcubic connected graph of order $n$. Suppose first that $G$ is triangle-free. If $G$ is not 3-regular, we first run the $O\left(n^{4}\right)$ algorithm of Fraughnaugh and Locke [12], which finds an independent set $W$ of size at least $11 n / 30-1 / 30$ in $G$. Then the set

$$
S:=V \backslash W, \quad|S| \leq 19 n / 30+1 / 30
$$

meets all edges of $G$ and hence is a clique-transversal set of required size, found in polynomial time. If $G$ is triangle-free and cubic, then the algorithm in [12] guarantees a slightly weaker lower bound $|W| \geq$ $11 n / 30-2 / 15$ on the size of independent set $W$, and we obtain $|S| \leq 19 n / 30+2 / 15$ in this case.

Suppose from now on that $G$ contains a triangle, say $T$ with vertex set $\left\{x_{1}, x_{2}, x_{3}\right\}$. Each $x_{i} \in T$ $(i=1,2,3)$ has at most one neighbor outside $T$. We assume $d\left(x_{1}\right) \geq d\left(x_{2}\right) \geq d\left(x_{3}\right)$, and if $d\left(x_{i}\right)=3$ then denote the neighbor of $x_{i}$ outside $T$ by $y_{i}$.

If $d\left(x_{1}\right)=2$, then $G \simeq K_{3}$; and if $d\left(x_{3}\right)=3$ and $y_{1}=y_{2}=y_{3}$, then $G \simeq K_{4}$. In either case, $\tau_{C}(G)=1 \leq n / 3$ holds, and we have nothing to prove. Similarly, it is easy to check that $\tau_{C}(G) \leq n / 2$ is valid if $n \leq 4$. Hence, we assume $d\left(x_{1}\right)=3$ and $n>4$.

We shall apply induction on $n$, assuming that the upper bound $\tau_{C}\left(G^{\prime}\right) \leq 19 n^{\prime} / 30+1 / 30$ is valid for all non-cubic $G^{\prime} \in \mathcal{G}_{3}$ of order $n^{\prime}<n$. For disconnected subcubic graphs with $K$ components, none of which is cubic, this equivalently means $\tau_{C}\left(G^{\prime}\right) \leq 19 n^{\prime} / 30+K / 30$. Note that no proper subgraph of $G$ can have cubic components, because $G$ is connected. The following simple fact will also be useful. 
Remark 1 Removing any set $U$ of vertices, the number of components in the remaining graph cannot be larger than the edges connecting $U$ with $V \backslash U$.

We now proceed with the inductive step for the upper bound on $\tau_{C}$. If $d\left(x_{2}\right)=2$, then $G-T$ is connected and it has a clique-transversal set $S^{\prime}$ of size at most $19(n-3) / 30+1 / 30$ by the induction hypothesis. Since $S:=S^{\prime} \cup\left\{x_{1}\right\}$ is a clique-transversal set in $G$, the upper bound $\tau_{C}(G) \leq 19 n / 30-$ $13 / 15$ follows.

Suppose $d\left(x_{2}\right)=3$ and $y_{1} \neq y_{2}$. If $d\left(x_{3}\right)=2$, or $d\left(x_{3}\right)=3$ but $y_{3}=y_{1}$ (or $y_{3}=y_{2}$ ), we consider the graph $G-T-y_{1}$ (or $\left.G-T-y_{2}\right)$. Since it has at most three connected components by Remark 1 . it contains a clique-transversal set $S^{\prime}$ of size at most $19(n-4) / 30+3 / 30$, and then $S:=S^{\prime} \cup\left\{y_{1}, x_{2}\right\}$ meets all cliques of $G$. Thus, $\tau_{C}(G) \leq 19 n / 30+(3-76+60) / 30=19 n / 30-13 / 30$.

Finally, suppose $d\left(x_{3}\right)=3$ and $y_{1} \neq y_{2} \neq y_{3} \neq y_{1}$. We now consider $G-T-y_{1}-y_{2}$. By Remark 1 it has at most five connected components. Hence, by the induction hypothesis, it has a clique-transversal set $S^{\prime}$ of size at most $19(n-5) / 30+5 / 30$, and $S:=S^{\prime} \cup\left\{y_{1}, y_{2}, x_{3}\right\}$ is a clique-transversal set in $G$. Thus, $\tau_{C}(G) \leq 19 n / 30+(5-95+90) / 30=19 n / 30$.

Time analysis. Let us choose a polynomial $P(x)$ satisfying the following properties: $P(x)$ is monotone increasing for $x>0, P(n)$ is an upper bound for all $n$ on the running time of the $O\left(n^{4}\right)$ algorithm in [12] for triangle-free subcubic graphs, moreover

$$
P\left(x^{\prime}\right)+P\left(x^{\prime \prime}\right) \leq P\left(x^{\prime}+x^{\prime \prime}\right) \quad \text { and } \quad P(x-3)+c x \leq P(x)
$$

for all $x^{\prime}, x^{\prime \prime} \geq 1$, all $x \geq 4$, and for some constant $c$ to be fixed later. For instance, if $\sum_{i=0}^{4} a_{i} x^{i}$ is a valid bound for [12], then $P(x):=\sum_{i=0}^{4}\left|a_{i}\right| x^{i}+c x^{2}$ will do; and any faster algorithm for triangle-free graphs would yield a stronger estimate for the general case, too.

If $G$ is triangle-free, then the algorithm terminates in at most $P(n)$ steps by assumption. Otherwise, triangle $T$ can be found in $c_{1} n$ steps for some constant $c_{1}$, e.g. applying breadth-first search and checking at each vertex whether its two descendants (or possibly three for the root vertex) are adjacent or not.

The removal of 3,4 , or 5 vertices takes constant time. Assuming that the remaining graph has connected components of orders $n_{1}, \ldots, n_{k}$, we need at most $c_{2}\left(n_{1}+\ldots+n_{k}\right)$ steps to determine its components and at most $P\left(n_{1}\right)+\ldots+P\left(n_{k}\right) \leq P\left(n_{1}+\ldots+n_{k}\right) \leq P(n-3)$ steps to find the partial cliquetransversal set $S^{\prime}$. In this way, choosing $c=c_{1}+c_{2}$ we obtain that $P(n)$ is an upper bound on the total running time.

\section{Weak 2-coloring}

In this section we prove Theorem 2. Since even cycles are trivial to 2-color, we assume that $G$ is not a cycle. It will turn out that diamond-free graphs admit a more elegant approach than general ones, therefore we treat them first; and afterwards the idea will be to identify a diamond $D$, find a weak 2-coloring of $G-D$, and prove that it can be extended to a weak 2-coloring of $G$.

So, assume first that $G$ is connected, claw-free and also diamond-free, has maximum degree at most four, and is not a chordless cycle of length greater than three. Under these conditions we say that $G$ is a safe graph. Moreover, Let us call a vertex $x$ safe if it satisfies the following requirements:

1. $G-x$ is connected,

2. $G-x$ is not a cycle longer than three, 
3. $x$ is either a pendant vertex or contained in a $K_{3} \subseteq G$.

For a safe vertex $x$ we define its critical neighbor $y$-whose choice is not always unique-as follows.

- If $d(x)=1$, then $y$ is the unique neighbor of $x$.

- If $x$ is in some triangle $T_{x}$, let $K_{x}$ be the (unique) clique containing $T_{x}$.

- If $x$ has neighbor(s) outside $K_{x}$, let $y \notin K_{x}$ be any such neighbor.

- Otherwise, let $y \in K_{x}$ be any neighbor of $x$.

Note that $K_{x}$ is well-defined because each edge (and hence also each triangle) of $G$ lies in a unique clique, otherwise $G$ would not be diamond-free. For the same reason, $x$ cannot occur in two triangle cliques which share a further vertex. And $x$ cannot be involved in two cliques of size two either, because they would induce a claw with a vertex of $T_{x}$. On the other hand, it can happen that $x$ is incident with two edge-disjoint triangles, in this case $T_{x}=K_{x}$ can be chosen as any one of them.

We proceed with some properties concerning safe vertices in safe graphs.

Lemma 1 If $x$ is a safe vertex in a safe graph $G$, and $x$ is contained in a triangle $T_{x}$, then also $K_{x}-x$ is a clique in $G-x$ for the unique clique $K_{x}$ containing $T_{x}$ in $G$.

Proof: Otherwise, there is a vertex $z$ adjacent to all vertices of $K_{x}$. In this case, $x y$ must be a non-edge, by the maximality of $K_{x}$. But then $T_{x} \cup\{z\}$ induces a diamond, a contradiction.

Lemma 2 Every safe graph of order greater than one has a safe vertex.

Proof: Let $G$ be a safe graph. Suppose first that $G$ has a leaf $x$. The only safe-vertex-defining condition which could be violated is 2 , but then we would find a claw in $G$. Thus, $x$ is safe.

Assume next that $G$ has no pendant vertices. Then $G$ is not a tree, and it contains a chordless cycle. If this cycle can be chosen with length at least four, then we denote it by $C$. Since $G$ is not a cycle, there exists some vertex $u$ adjacent to $C$. Claw-freeness implies that there is an edge $e=x y$ in $C$ such that $x y u$ is a triangle. If $G-x$ is disconnected, then the two neighbors of $x$ on $C$ and a third neighbor in another component of $G-x$ form a claw with center $x$. Hence, $G-x$ has to be connected, and again it suffices to check whether Condition 2 is valid.

Suppose on the contrary that the graph $G-x$ is a chordless cycle. Let $z \neq y$ be the other neighbor of $x$ on $C$. In this case, $G-x$ consists of two paths, namely $P:=C-x$ from $y$ to $z$ and a $z-u$ path $Q$, completed to a chordless cycle with edge $u y$. The neighbors of $x$ are $y, u, z$, and the neighbor of $z$ on $Q$. This is the only situation where $x$ violates Condition 2. But then both $y$ and $z$ are safe in $G$.

Finally, if $G$ has no chordless cycles of length at least four, then $G$ is chordal, by definition. It is a well-known fact that a chordal graph has a simplicial vertex $x$, which clearly is safe.

Lemma 3 Let $x$ be a safe vertex in a safe graph $G=(V, E)$, with critical neighbor $y$. If $\phi: V \backslash\{x\} \rightarrow$ $\{r, g\}$ is a weak 2-coloring of $G-x$, then $\phi(x):=\{r, g\} \backslash\{\phi(y)\}$ extends it to a weak 2-coloring of $G$.

Proof: Suppose on the contrary that some monochromatic clique $R$ occurs in $G$, say completely red. Of course, $x \in R$ and $|R| \geq 2$. Let $W$ be the complete subgraph $R-x$. This $W$ is not maximal in $G-x$ 
since $\phi$ is a weak 2-coloring of $G-x$. Hence, By Lemma 1 we have $|W|=1$, say $W=\{w\}$. Note that $w \neq y$ because $\phi(w)=\phi(x) \neq \phi(y)$.

Vertex $x$ is not pendant, therefore its $K_{x}$ is well-defined. Since the edge $w x$ is a clique in $G$ and so it cannot be contained in any triangle, we see that $w y \notin E$, moreover $w$ is not in $K_{x}-x$.

By Lemma 1, $K_{x}-x$ is a clique in $G-x$, consequently both $y$ and $w$ have some non-neighbors in $K_{x}-x$; denote one non-neighbor by $y^{\prime}$ and $w^{\prime}$, respectively. Then $y w^{\prime}$ is an edge, otherwise $\left\{x, y, w, w^{\prime}\right\}$ would induce a claw. But now $y y^{\prime} \notin E$ implies $y^{\prime} \neq w^{\prime}$ and that $\left\{x, y, y^{\prime}, w^{\prime}\right\}$ induces a diamond, a contradiction.

Based on these lemmas, we design Algorithm[1] as a subroutine for the general algorithm to find a weak 2-coloring.

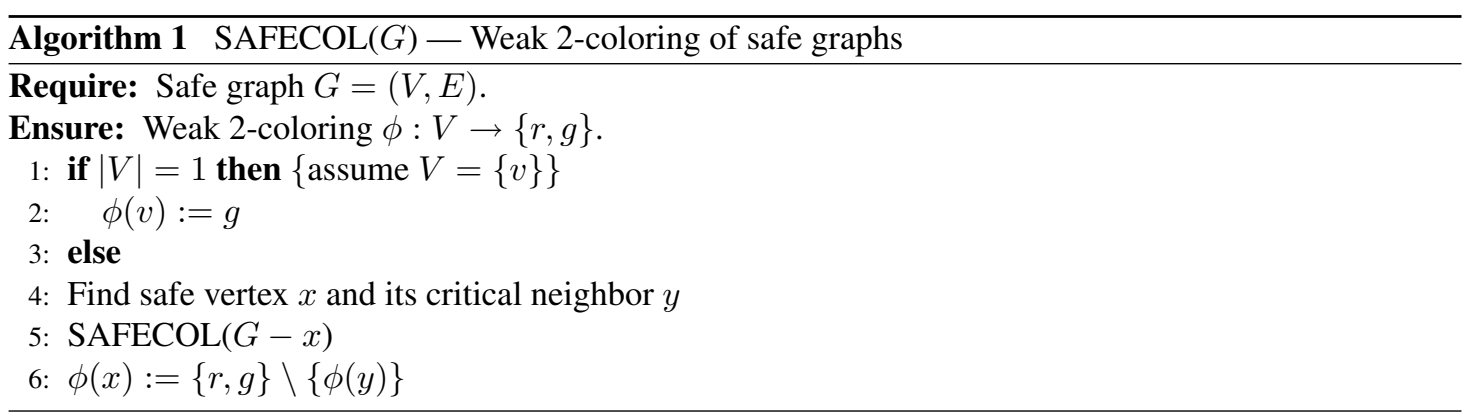

Time analysis for Algorithm 1. Apart from the recursive call in Step 5, the only time-consuming instruction is to identify a safe vertex in Step 4. Efficient implementation is ensured by the following claim.

Lemma 4 A safe vertex in a safe graph can be found in linear time.

Proof: The non-cutting vertices $x$ of $G$ can be enumerated in $O(n)$ steps, and since $G$ has bounded maximum degree (and also because it is claw-free), for each $x$ it can be tested in constant time whether or not $x$ is incident with a triangle. Finally, $G-x$ can be a cycle for at most one choice of $x$.

Hence, storing the eliminated vertices in a stack, the recursive call of Step 5 (which yields iterated executions of Steps 4 and 6) can be implemented efficiently. As a consequence, Algorithm 1 requires not more than $O\left(n^{2}\right)$ steps.

The following side-product of our method appears to be of interest on its own right, too.

Remark 2 Since every subgraph of any safe $G \nsucceq K_{1}$ contains a safe vertex, a "safe elimination order" can be determined.

From now on we suppose that $G$ contains a diamond $D \simeq K_{4}-e$. Some cliques of $G$ have vertices in both $D$ and $G-D$; we call them crossing cliques. If a crossing clique $Q$ has just one vertex in $D$, we say that $Q$ is a strong crossing clique; and otherwise we say that $Q$ is weak.

As for notation, we assume that the diamond $D$ found in $G$ has vertex set $\left\{c_{1}, c_{2}, d_{1}, d_{2}\right\}$, where the only non-edge is $\left\{c_{1}, c_{2}\right\}$. By the degree assumption, there can occur at most one edge from $d_{i}$ to $M:=G-D$, and at most two edges from $c_{i}$ to $M(i=1,2)$. Due to these degree constraints and the assumption that $G$ is claw-free, combinations of the following crossing cliques may occur: 
- strong edge: $c_{i} a_{i}$ (at most one for each $i \in\{1,2\}$ )

- strong triangle: $c_{i} b_{i}^{\prime} b_{i}^{\prime \prime}$ (at most one for each $i \in\{1,2\}$ )

- weak triangle: $c_{i} d_{j} w_{i, j}$ (at most one for each pair $(i, j)$ )

- weak 4-clique: $c_{i} d_{1} d_{2} z_{i}$ (at most one for each $i \in\{1,2\}$ )

Degree bounds on $d_{1}, d_{2}$ imply that if both $w_{1, j}, w_{2, j}$ exist, then $w_{1, j}=w_{2, j}$; and similarly, if both $z_{1}, z_{2}$ exist, then $z_{1}=z_{2}$. Moreover, weak triangles of type $d_{1} d_{2} v$ would create a claw, hence are excluded.

The procedure can now be formalized as described in Algorithm 2. The heart of the proof is expressed in the following assertion.

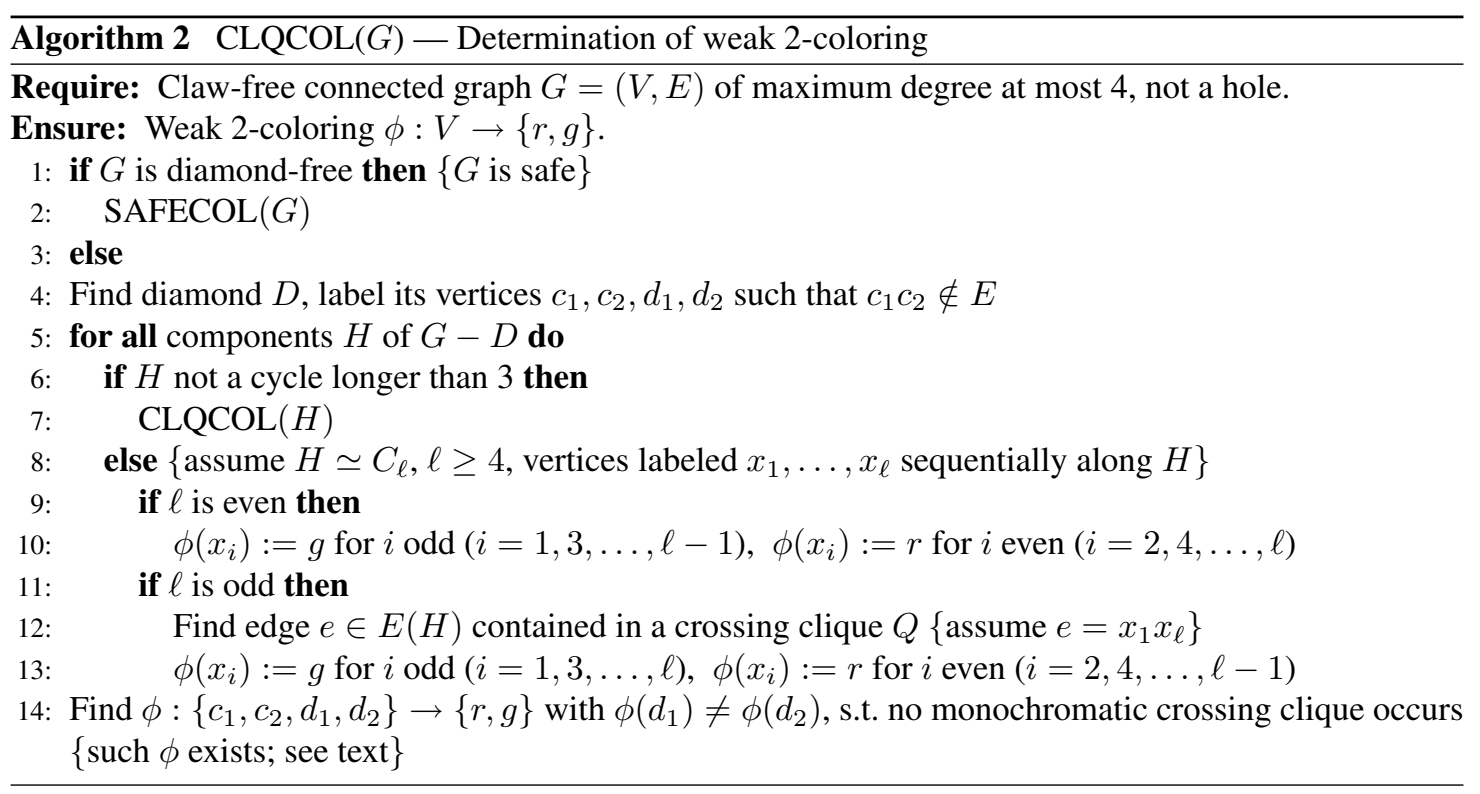

Lemma 5 Let $G \in \mathcal{G}_{4}$ be claw-free, and $D$ a diamond in $G$. If no component of $G-D$ is an odd hole, then every weak 2-coloring of $G-D$ can be extended to a weak 2-coloring of $G$ in such a way that the two vertices of degree three inside $D$ get distinct colors.

Proof: Suppose that a weak 2-coloring $\phi$ of $G-D$ has been fixed. We wish to extend it to the entire $G$ without changing any color in $G-D$; the extension will also be denoted by $\phi$.

Once we decide that $\phi\left(d_{1}\right) \neq \phi\left(d_{2}\right)$ holds, all cliques of $G$ with three vertices in $D$ are 2-colored. This includes the triangles of $D$ and the weak 4-cliques, too, if there are any. Therefore, we only have to show that the crossing cliques of orders two and three-strong edge, strong triangle, weak triangle - are 2-colorable under this condition.

A strong crossing clique may determine the color of $c_{i}$. Namely, $\phi\left(c_{i}\right)=\{r, g\} \backslash\left\{\phi\left(a_{i}\right)\right\}$ must hold in a strong edge, and likewise, $\phi\left(b_{i}^{\prime}\right)=\phi\left(b_{i}^{\prime \prime}\right)$ in a strong triangle forces $\phi\left(c_{i}\right)=\{r, g\} \backslash\left\{\phi\left(b_{i}^{\prime}\right)\right\}$. Since each $c_{i}$ is incident with at most one strong clique, two contradictory conditions of this kind cannot occur at $c_{i}$. Moreover, apart from these situations, we have no a priori restriction on the colors of $c_{1}$ and $c_{2}$. 
Suppose first that $c_{1} a_{1}$ is a strong edge. Then $c_{1}$ cannot be incident with any crossing triangles: a strong one is impossible by the degree condition, and a weak triangle $c_{1} d_{1} w_{1,1}$ would create a claw on $\left\{c_{1}, d_{2}, a_{1}, w_{1,1}\right\}$ because $c_{1} a_{1}$ is a clique and hence $a_{1}$ cannot be adjacent to any neighbor of $c_{1}$. Consequently, $\phi\left(c_{1}\right):=\{r, g\} \backslash\left\{\phi\left(a_{1}\right)\right\}$ yields a 2-coloring for all crossing cliques incident with $c_{1}$. The same argument applies if there is a strong edge $c_{2} a_{2}$.

The situation is similar and only slightly more complicated if there is a strong triangle, say $c_{1} b_{1}^{\prime} b_{1}^{\prime \prime}$. In this case further edges $b_{1}^{\prime} d_{1}$ and/or $b_{1}^{\prime \prime} d_{2}$ may be present, creating one or two weak triangles (or weak 4-cliques). If $\phi\left(b_{1}^{\prime}\right)=\phi\left(b_{1}^{\prime \prime}\right)$, the choice $\phi\left(c_{i}\right):=\{r, g\} \backslash\left\{\phi\left(b_{1}^{\prime}\right)\right\}$ 2-colors those weak triangles as well, and the proof is done. On the other hand, if $\phi\left(b_{1}^{\prime}\right) \neq \phi\left(b_{1}^{\prime \prime}\right)$, then we may disregard the strong triangle because it is already 2-colored, independently of the actual color of $c_{2}$.

From now on we may assume that $c_{1}$ and $c_{2}$ are contained in weak triangles only. We select one $c_{1} d_{i} w_{1, i}$ and one $c_{2} d_{j} w_{2, j}$, and define $\phi\left(c_{1}\right):=\{r, g\} \backslash\left\{\phi\left(w_{1, i}\right)\right\}, \phi\left(c_{2}\right):=\{r, g\} \backslash\left\{\phi\left(w_{2, j}\right)\right\}$. This leaves at most one monochromatic weak triangle on each of $c_{1}$ and $c_{2}$. If such a triangle remains on one of $c_{1}$ and $c_{2}$ only, then some of $\left(\phi\left(d_{1}\right), \phi\left(d_{2}\right)\right):=(g, r)$ and $\left(\phi\left(d_{1}\right), \phi\left(d_{2}\right)\right):=(r, g)$ surely makes it 2colored. In the other case both $d_{1}$ and $d_{2}$ occur in two weak triangles; but each $d_{i}$ has only one neighbor in $G-D$, therefore we must have $w_{1,1}=w_{2,1} \neq w_{1,2}=w_{2,2}$. Here $w_{2,1} \neq w_{1,2}$ holds because otherwise two weak 4-cliques would occur instead of four weak triangles.

If $\phi\left(w_{2,1}\right) \neq \phi\left(w_{1,2}\right)$, a simple completion of the coloring is to put $\phi\left(d_{1}\right):=\phi\left(w_{1,2}\right)$ and $\phi\left(d_{2}\right):=$ $\phi\left(w_{2,1}\right)$; and if $\phi\left(w_{2,1}\right)=\phi\left(w_{1,2}\right)$, then all the four weak triangles have a vertex of opposite color at $c_{1}$ or $c_{2}$, and we obtain a weak 2 -coloring by assigning $\left(\phi\left(d_{1}\right), \phi\left(d_{2}\right)\right):=(g, r)$.

Based on Lemma 5 , the soundness of Algorithm 2 can be verified easily, although it needs a little case distinction because odd hole components in $G-D$ are not weakly 2-colorable. If a component $H \not K_{3}$ of $G-D$ is an odd cycle longer than three, however, then any edge connecting $H$ with $D$ has to be extendable to a triangle with two vertices in $H$, for otherwise a claw would occur. Hence, edge $e$ in Step 10 is well-defined, and it induces a strong triangle with $c_{1}$ or $c_{2}$. That is, the situation is the same as if the strong triangle occurred from a non-cycle component, and the argument given in the proof of Lemma 5 verifies that all crossing cliques are 2-colored.

Time analysis for Algorithm 2. As it has been shown, Algorithm 1 called in Step 2 runs within $c n_{i}^{2}$ time on any graph of order $n_{i}$, for some absolute constant $c$. Observe further that, no matter how many times it is performed during the recursive calls of Step 7, the safe subgraphs occurring in the procedure are mutually vertex-disjoint. Consequently, the overall running time of this part of Algorithm 2 does not exceed $c n^{2}$.

Even better, cycles in Steps 8-13 need time proportional to $\ell$, and also those cycles are mutually vertexdisjoint. Hence, they require $O(n)$ time altogether. Also, Step 14 requires constant time for $D$, because only few crossing cliques can occur and they can be enumerated in constant time. These constants sum up to $O(n)$ through all iterations.

Since the vertex degrees are bounded, we need at most $c^{\prime} n$ time to determine diamond D in Step 4. Also, we can enumerate the components of $G-D$ in Step 5 and check the condition in Step 6 in linear time. Hence, reduction to a smaller problem instance takes linear time. Thus, the overall running time of the algorithm is $O\left(n^{2}\right)$. 


\section{Concluding remarks}

Here we put a couple of simple observations and mention some problems, which would be of interest for future research.

NP-completeness. From the well-known fact that the independence number is NP-complete to determine on cubic graphs, in connection with Theorem 1 we can derive that the complexity of finding $\tau_{C}$ is NP-complete on triangle-free cubic graphs. The proof can be done in two steps:

- Given a cubic graph $G=(V, E)$, replace each edge $e=x y \in E$ by a path $x v_{e} w_{e} y$ of length three. This operation yields a subcubic triangle-free graph $H$, and increases the independence number by exactly $|E|$.

- Take two copies $H^{\prime}, H^{\prime \prime}$ of $H$ and insert the edges $v_{e}^{\prime} w_{e}^{\prime \prime}$ and $v_{e}^{\prime \prime} w_{e}^{\prime}$ for all $e \in E$. This results in a cubic triangle-free graph whose independence number is the double of that in $H$.

Optimum running time. Although our algorithms run in polynomial time, we expect that the orders of those polynomials are not optimal. For this reason, it is natural to ask:

Problem 1 Determine the best asymptotic running time of an algorithm for

1. finding clique-transversal sets of size at most $19 n / 30+O(1)$ in connected subcubic graphs,

2. finding weak 2-colorings in claw-free graphs of maximum degree four.

Clique-transversal number vs. clique size. The flavor of results in [22] is that if every edge of a 'nicely structured' graph lies in a 'large' clique, then $\tau_{C}$ is 'small'. This direction has been pursued in [3] and recently in [5]. We think that there are many further classes of graphs for which such kind of results would be of interest to study.

Line graphs. The line graph of $K_{6}$ is 8 -regular and is not weakly 2-colorable. This fact, together with our Theorem 2, leads to the following problem.

Problem 2 Find the largest integer $d$ such that every claw-free graph of maximum degree $d$ is weakly 2-colorable.

Perfect graphs. A long-standing open problem of Duffus et al. [10] asks whether $\chi_{C}$ is bounded above by a constant on the class of perfect graphs. In fact, no examples of perfect graphs $G$ with $\chi_{C}(G)>3$ are known. The upper bound $\chi_{C} \leq 3$ has been proved for some classes of perfect graphs in [6]. Moreover, it is immediate by definition that every strongly perfect graph is weakly 2-colorable.

\section{References}

[1] M. Aigner and Th. Andreae, Vertex-sets that meet all maximal cliques of a graph, manuscript, 1986.

[2] Th. Andreae, On the clique-transversal number of chordal graphs, Discrete Math. 191 (1998), 3-11.

[3] Th. Andreae and C. Flotow, On covering all cliques of a chordal graph, Discrete Math. 149 (1996), 299-302. 
[4] Th. Andreae, M. Schughart, and Zs. Tuza, Clique-transversal sets of line graphs and complements of line graphs, Discrete Math. 88 (1991), 11-20.

[5] S. Aparna Lakshmanan and A. Vijayakumar, The $\langle t\rangle$-property of some classes of graphs, Discrete Math. 309 (2009), 259-263.

[6] G. Bacsó, S. Gravier, A. Gyárfás, M. Presissmann, and A. Sebő, Coloring the maximal cliques of graphs, SIAM J. Discrete Math. 17 (2004), 361-376.

[7] V. Balachandhran, P. Nagavamsi, and C. Pandu Rangan, Clique transversal and clique independence on comparability graphs, Inform. Process. Lett. 58 (1996), 181-184.

[8] M. S. Chang, Y. H. Chen, G. J. Chang, and J. H. Yan, Algorithmic aspects of the generalized cliquetransversal problem on chordal graphs, Discrete Appl. Math. 66 (1996), 189-203.

[9] G. J. Chang, M. Farber and Zs. Tuza, Algorithmic aspects of neighbourhood numbers, SIAM J. Discrete Math. 6 (1993), 24-29.

[10] D. Duffus, B. Sands, N. Sauer, and R. E. Woodrow, Two-coloring all two-element maximal antichains, J. Combin. Theory A 57 (1991), 109-116.

[11] P. Erdős, T. Gallai, and Zs. Tuza, Covering the cliques of a graph with vertices, Discrete Math. 108 (1992), 279-289.

[12] K. Fraughnaugh and S. C. Locke, 11/30 (Finding large independent sets in connected triangle-free 3-regular graphs), J. Combin. Theory B 65 (1995), 51-72.

[13] T. Gallai, unpublished, mid-1980's.

[14] V. Guruswami and C. Pandu Rangan, Algorithmic aspects of clique-transversal and cliqueindependent sets, Discrete Appl. Math. 100 (2000), 183-202.

[15] J. Kratochvíl and Zs. Tuza, On the complexity of bicoloring clique hypergraphs of graphs. J. Algorithms 45 (2002), 40-54.

[16] C. M. Lee and M. S. Chang, Distance-hereditary graphs are clique-perfect, Discrete Appl. Math. 154 (2006), 525-536.

[17] Z. Liang, E. Shan, and T. C. E. Cheng, Clique-transversal sets in cubic graphs, in B. Chen, M. Paterson, and G. Zhang (Eds.): ESCAPE 2007, LNCS 4614, Springer-Verlag, pp. 107-115, 2007.

[18] S. C. Locke, Bipartite density and the independence ratio, J. Graph Theory 10 (1986), 47-53.

[19] D. Marx, Complexity of clique coloring and related problems, to appear. (Manuscript, 2004.)

[20] B. Mohar and R. Škrekovski, The Grötzsch theorem for the hypergraph of maximal cliques, Electr. J. Combin. 6 (1999), R26.

[21] E. Shan, T. C. E. Cheng, and L. Kang, Bounds on the clique-transversal number of regular graphs, Science in China Ser. A: Math. 51:5 (2008), 851-863.

[22] Zs. Tuza, Covering all cliques of a graph, Discrete Math. 86 (1990), 117-126. 